REDES- Revista hispana para el análisis de redes sociales

Vol.20,\#11a, Junio 2011

http://revista-redes.rediris.es

\title{
Alianzas y redes de parentesco de gitanos en Cataluña ${ }^{1}$
}

\author{
Missaoui Hasnia-Sonia - Profesora Titular en Sociología - \\ Université Toulouse II Le Mirail, LISST-CERS, UMR 5193, CNRS, UTM y EHESS
}

\begin{abstract}
Resumen
A partir de un trabajo de tesis centrado en la « experiencia de la diversidad social vivida » de los niños gitanos sedentarios o en migración y de los niños marroquíes de familias que han inmigrado recientemente a Toulouse, Perpiñán y Barcelona, hemos tratado de identificar los procesos de abandono escolar de dichos niños en los contextos comunitarios en que la escuela no puede asegurarles, por si misma, la transmisión de las competencias culturales y sociales para su autonomía adulta y ciudadana. El análisis se centra en las interacciones que ligan estos dos espacios de socialización: la familia y la escuela, y en las competencias de los jóvenes niños provenientes de medios socio-económicos desfavorecidos, para atravesar estos distintos mundos de socialización. Para este artículo, presentaremos particularmente dos trayectorias intergeneracionales de familias gitanas catalanas que nos han permitido comprender cómo ciertas familias podían preservar la transmisión de competencias prácticas (savoir faire) y de aprendizajes escolares. A partir de estos trazados genealógicos veremos cómo la familia gitana de Perpiñán y la familia gitana barcelonesa, se refuerzan mutuamente aglomerándose en un clan de nuevos contornos, transnacionales, cuando una lectura limitada por la frontera política entre Francia y España nos sugeriría la desagregación, a veces incluso simultánea, de una y otra.
\end{abstract}

Palabras clave: Gitanos, movilidad transfronteriza, tiempo inter-generacional, autoformación, autonomía social.

\begin{abstract}
Some characteristics of new migrants issued from Moroccan and settled gipsy communities are studied. It is demonstrated how they develop skills to be "here and there" based on a know-how for international travel, hence creating new models of identification relying on experiences of multiple interaction. These new types of migrants are highly mobile and produce micro-societies with singular norms with new adapted social interactions that transform the concerned institutions: school, family and economic processes. The study of genealogical lines indicates us how Barcelona and Perpignan Gypsy families reinforce each other by aggregating themselves into clans with new transnational outlines, whereas by contrast a reading limited by Franco-Spanish political borders suggests the disintegration of one and sometimes of both simultaneously. Those genealogical lines help bring to light material and symbolic spaces, that have led me to locate and analyse a new form of social autonomy.
\end{abstract}

Key words: Gypsies, cross-border mobility, intergenerational time, self-training, social autonomy.

\footnotetext{
${ }^{1}$ Artículo traducido por Ainhoa de Federico de la Rúa e Irene Cruz.
} 


\section{Introducción}

En este artículo hablaremos del fenómeno transfronterizo, fenómeno que no es nuevo pero que se convierte en transversal a nuestras temáticas de estudio y por ello a nuestro cuestionamiento sociológico. Preguntarse hoy en día sobre lo que produce cohesión social significa preguntarse por las nuevas formas migratorias, por las nuevas figuras identitarias que se derivan de ellas y por las redes (o lazos) que las identifican. Una de las dificultades parece ser nuestra capacidad a captar este nuevo "actor" que circula, se instala y a veces sólo está de paso transformando nuestras sociedades y sus prácticas. Este actor, siempre designado por su exterioridad, y sin embargo igualmente actor-ciudadano, no es fácilmente visible y actúa a menudo de forma discreta para construirse una vida mejor. Nuestro trabajo nos ha permitido captar mejor las competencias e iniciativas de las poblaciones frecuentemente designadas como "Otros" y siempre a distancia del "Nosotros" colectivo.

Nuestro trabajo se apoya en las investigaciones llevadas a cabo por Alain Tarrius quien, desde hace ya bastantes años, insiste en las competencias de movilidad de ciertos migrantes internacionales (en particular aquellos que van y vienen entre su país de acogida y sus países de origen) y en su participación a la mundialización como si fuesen "hormigas"2". Aparecen así otras formas más complejas de economía que movilizan redes de relaciones (redes familiares o de compadres) y de competencias prácticas (savoir faire) marcadas por su competencia en la transnacionalidad. Estas nuevas formas de la economía se emparentan con un vasto dispositivo de economía sumergida de intercambios de mercancías entre Europa, el Magreb, el África subsahariana, el próximo y el mediano Oriente. Estas "hormigas" de la "mundialización por abajo"3 invierten el esquema de la construcción identitaria, imponiendo a la clásica oposición entre los nuestros y los suyos, entre ser de aquí o de allí, otra forma triádica: ser de aquí, ser de allí y ser de aquí y de allí a la vez .

\footnotetext{
2 Tarrius Alain, (1992), Les fourmis d'Europe : migrants riches, migrants pauvres et nouvelles villes internationales. L' Harmattan. 210 p, Paris.

3 Tarrius Alain, (2002), La mondialisation par le bas. Les nouveaux nomades des économies souterraines. Balland. 169 p. 2002, Paris.

4 Missaoui Lamia., Tarrius Alain, (2004), «Parole, honneur, mobilité. Les " petits migrants " dans les réseaux mondiaux de l'économie souterraine », In Economies Choisies, Dir. N. Barbe et S. Latouche, Colección Ethnologie de la France, Cahier 20, Ed. de la Maison des Sciences de I'Homme. Véase también Missaoui Lamia, (1995), «Généralisation du commerce transfrontalier : petit ici, notable là-bas », REMI, Volume 11, p. 53-75.
} 


\section{De la cuestión del abandono escolar ${ }^{5}$ a la cuestión de las redes familiares...}

En nuestro trabajo ${ }^{6}$, hemos tratado de identificar los procesos de abandono escolar de hijos de gitanos y de marroquíes de reciente inmigración, en los contextos comunitarios en que la escuela no puede asegurarles, ella sola, la transmisión de competencias culturales y sociales, con sus efectos económicos, requeridos en nuestra sociedad para adquirir la autonomía adulta y ciudadana. Durante nuestras investigaciones hemos identificado estos procesos, no tanto en una u otra de las instituciones escolares o familiares, sino en las interacciones que ligan ambos espacios de socialización. Nuestro trabajo se ha centrado, por lo tanto, en la experiencia de "diversidad social vivida" de los niños gitanos sedentarios o en migración y de los niños marroquíes de familias de reciente inmigración a Toulouse, Perpiñán o Barcelona. Diversas observaciones nos habían conducido a notar que, en medio de la abandono escolar generalizado y notorio de dichos jóvenes constatado tantas veces, se producían algunas trayectorias de éxito escolar en la primaria y la secundaria. Estábamos sensibilizados al hecho de que los jóvenes encontrados vivían diferentes formas de mestizaje como consecuencia de los grandes trayectos migratorios o de las transformaciones de los modos de vida comunitarios.

Esta experiencia contemporánea de una transformación de los universos de normas, en el sentido de una intensificación de las mezclas de las poblaciones y las trayectorias cosmopolitas, no era suficiente para apuntalar nuestras hipótesis de investigación. Si actualmente observamos un movimiento de afirmación comunitaria entre las poblaciones gitanas, no se trata de las formas sociales y económicas que caracterizan a los nuevos inmigrantes marroquíes.

\section{a) Las prácticas de las familias y los niños de familias magrebíes de reciente inmigración}

En efecto, las familias marroquíes de reciente inmigración están generalmente incluidas en redes familiares dispersas a escala europea, que se constituyen cada vez más frecuentemente como redes económicas transnacionales y asumen roles de "hormigas" de las economías sumergidas de productos de uso lícito entre Europa y

\footnotetext{
5 Hasnia-Sonia Missaoui, coll. Alain Tarrius, (2004), « L'auto-formation communautaire des jeunes gitans et marocains », en La Déscolarisation bajo la dirección de Dominique Glasman y Françoise Oeuvrard, Édition La Dispute, 15 p.

6 Missaoui Hasnia-Sonia, (2005), Thesis en Sociología - De l'étranger au citoyen : l'école déstabilisée. Mixités scolaires, mixités familiales et attitudes face à la déscolarisation d'enfants Gitans et Maghrébins, Departamento de Sociologie, Universidad de Toulouse 2 - Le Mirail, y Centre d'Etudes des Rationalités et des Savoirs (CIRUS-CERS UMR 5193), Toulouse.
} 
el Magreb. Esta especie de concepción de la mundialización de los intercambios a una forma, "por abajo", de capitalismo nómada se ve acompañada de un cuestionamiento del encuentro cara a cara entre extranjeros y Estados-nación depositarios del esquema clásico de la integración: estos nuevos migrantes-actores, que saben reducir las fronteras de la etnicidad (en sus intercambios comerciales con todo tipo de poblaciones sin distinción) refuerzan de hecho su pertenencia a un lugar único de origen, Marruecos en el caso de las poblaciones estudiadas. Ya no se trata de las clásicas diásporas, que proveen rápidamente poblaciones económicamente complementarias de las sociedades de acogida. Esta forma migratoria original, que se amplifica año tras año, es la que hemos abordado en los juegos complejos de interacciones internas y externas que la caracterizan.

Los adolescentes de las familias marroquíes recientemente llegadas a Europa, abandonan masivamente la secundaria, según las modalidades comerciales de su familia, contrariamente a los hijos de la migración tradicional cuyas familias sedentarizadas apuestan por la integración únicamente a través del éxito escolar. Los padres de niños en clases de acogida han subrayado la necesidad de "saber apañarse" en la sociedad como un objetivo más importante que obtener un diploma. Estos adolescentes, desde los 13 o 14 años, son invitados por su padres a abandonar la secundaria en cuanto se presenta una oportunidad, en el entorno inmediato o en otra nación europea, de comenzar una actividad profesional en compañía de un pariente cercano o de un compadre de las redes de comercio. Recordemos que la mayoría de estos niños han pasado por una clase de acogida (clases especializadas en la escolarización de niños recién llegados) y no se hacen ilusiones sobre obtener el mínimo reconocimiento profesional gracias a un diploma cualquiera. Su futuro es tributario de la circulación de sus padres y de las redes sociales que los ligan. Los destinos de los jóvenes que han aceptado las reglas institucionales de la integración (aquellos nacidos en Francia) son rechazados por estas poblaciones recién llegadas: un diploma asociado con prácticas sin oportunidades y el paro mientras esperan la renta mínima de inserción ( $\mathrm{RMI}^{7}$ ) es considerado como la trayectoria paradigmática de la "vergüenza social".

\footnotetext{
7 NdT: el RMI o renta mínima de inserción es una ayuda del estado que puede recibir toda persona con la nacionalidad francesa (o de la UE) de más de 25 años y que no tenga otra fuente de ingresos declarada.
} 
Para estos magrebíes se podría decir que la verdadera escuela es la movilidad geográfica transnacional, un lugar en que conocer los medios sociales europeos, más que un lugar únicamente de aprendizaje de saberes ${ }^{8}$.

\section{b) Prácticas de las familias gitanas sedentarias y en circulación transfronteriza}

Entre las poblaciones gitanas contemporáneas, el cambio de los roles de las mujeres es el más llamativo. Éstas multiplican sus salidas de la comunidad, en particular con ocasión de lazos matrimoniales originales, por ejemplo con payos. Hemos constatado situaciones de diversidad familiar que sitúan al niño en el corazón de la construcción de nuevos proyectos, una forma de salvar y sacar adelante la familia nuclear. Otra constatación tiene que ver con los "sabios", los tíos, generalmente de una edad avanzada (55 a 70 años), que presentan capacidades operatorias constatadas en materia de lectura, de escritura, de cálculo. Estos tíos tienen un papel de transmisión de las competencias prácticas, concretamente a través de la autoformación de los adolescentes en el comercio". Estas formas de comercio se parecen a la "venta a la española"10 o a las ventas puerta a puerta. Los tíos y las mujeres (madres y abuelas) inician a los adolescentes a "saber apañarse", a adquirir competencias relacionales y sociales.

He aquí numerosas preguntas: ¿estaban las escuelas y los institutos mejor adaptados, al ser más diversos que las "escuelas gitanas" contemporáneas, hace varios decenios? ¿Conservarían mejor las familias sus competencias en sus linajes? ¿Cómo? ¿Por qué? ¿Existen en los clanes y familias, criterios y prácticas de designación de los jóvenes que son "dignos" de aprendizajes escolares?

Es importante recordar que ha existido una "edad de oro" económica relativamente reciente para numerosas familias gitanas catalanas que se puede situar en torno a los años 1930 tanto en Barcelona o Gerona en España, como en Perpiñán en Francia. Los oficios de trapero, de anticuario, de vendedor ambulante de obras de arte, aquellos asociados a los caballos, el chalaneo, la marroquinería en sillería y

\footnotetext{
8 Hasnia-Sonia Missaoui (2007.2-3), «Usage intermittent de l'école : des temporalités scolaires redéfinies par des enfants gitans et maghrébins nouveaux-arrivants », en «Les temporalités des populations », Espace, Populations, Sociétés, pp. 385-396.

9 Hasnia-Sonia Missaoui, (2005), L'école, le collège : y rester ou en sortir. La construction du potentiel de formation parmi les familles d'enfants gitans et maghrébins de Barcelone à Perpignan, Montpellier et Toulouse, Édition Trabucaire. Barcelone, Perpignan, 110 p.

10 NdT en Francia se llama « venta a la española » a la venta en la calle de objetos colocados en una manta atada con unas cuerdas y que se pueden retirar rápidamente cuando llega la policía.
} 
botería, y las presencias comerciales en los grandes mercados para la venta de telas han permitido a las familias, a menudo clanes, una movilización económica que se ha traducido, en concreto, por la compra de edificios burgueses en los barrios centrales de las ciudades (Barcelona, Gerona, Perpiñán). Este movimiento no duró mucho tiempo porque, en el lado español, el fascismo atacó rápidamente a estas grandes famillas, demasiado ligadas a la lengua y la cultura catalanas, combatidas por el poder franquista. La inmigración obligada de miles de gitanos andaluces miserables a los alrededores de Barcelona solicitó las solidaridades gitanas locales hasta comprometer la mayoría de los éxitos de los catalanes.

En Perpiñán, durante la segunda guerra mundial, la ocupación alemana mantuvo en una parálisis económica total a las familias asignadas a residencias en un barrio del centro urbano, St. Jaques. Luego, en los años 50, los oficios tradicionales en torno al caballo perdieron su razón de ser, es decir que los oficios de botería, sillería y chalaneo desaparecieron. Sin embargo algunas de estas familias, antes ricas, conservaron en buena parte de su patrimonio y sus oficios de trapero, anticuario y comerciante ambulante de tapices preciosos y de telas en los mercados semanales locales.

Nos parece útil desarrollar varias constataciones:

Primero, las situaciones de abandono escolar tienen poco sentido y poca importancia para las poblaciones en estudio. En suma, la integración por la escuela, la socialización ciudadana, es sinónimo de desagregación social para los gitanos y están sujetas a fuertes reticencias para los marroquíes recién llegados.

Segundo, en lo relativo a los gitanos catalanes, es necesario comprender cómo los oficios antes citados y las situaciones relativamente confortables de los que las ejercen han podido perpetuarse. Por ello llevamos a cabo una investigación sobre los éxitos profesionales de los gitanos a partir de la transmisión de las competencias prácticas antiguas, es decir las estrategias de reproducción internas a la comunidad, a las familias y a los clanes ${ }^{11}$ que la constituyen y que se reconocen en ella. Dicho de otro modo, se trata de una investigación sobre las relaciones familiares reactivadas o impulsadas por una mejor integración socio-económica.

11 Llamamos clan a los grupos formados por un cierto numéro de familias que comparten un antepasado común y que se reconocen en él. 
Además, una investigación ${ }^{12}$ en Perpiñán y en los pueblos de los Pirineos Orientales sobre los niveles de conocimientos de los gitanos según su edad, su sexo y su origen urbano o rural, confirma que el nivel escolar a descendido fuertemente en estas últimas décadas, en particular entre los chicos. Uno de los puntos a subrayar es que los hombres de más de 35 años aparecen como más instruidos que los jóvenes de 20, tanto en los medios rurales como los urbanos. Aquellos escolarizados en los pueblos obtienen mejores resultados que los que han frecuentado las escuelas de Perpiñán. Tras hacer esta constatación, nos pareció evidente que el mantenimiento de la diversidad en las escuelas de los pueblos explicaría las diferencias de resultados. A partir de ahí, hemos tratado de recoger las trayectorias intergeneracionales familiares para comprender mejor cómo ciertas familias podían preservar la transmisión de competencias prácticas y de los aprendizajes escolares.

Para este artículo $^{13}$, presentaremos particularmente dos trayectorias intergeneracionales familiares que nos han permitido comprender cómo ciertas familias podían preservar la transmisión de las competencias prácticas y de los aprendizajes escolares. A partir de estos trazados genealógicos veremos cómo la familia gitana Perpiñánesa y la familia gitana barcelonesa se refuerzan mutuamente aglomerándose en un clan con nuevos contornos transnacionales, mientras que una lectura limitada por la frontera política entre Francia y España nos sugiere la desagregación simultánea de una y otra.

Las reconstrucciones genealógicas han sido frecuentes en la antropología social, siendo ésta una de las bases para la emergencia del análisis de redes sociales (Scott 1991, Freeman 2004). Se puede considerar que los trazados genealógicos son un caso particular del análisis de redes de redes de parentesco.

\footnotetext{
12 Esta investigación ha sido realizada en colaboración con los investigadores Alain Tarrius y Lamia Missaoui y sobretodo con la ayuda de los profesores de las escuelas e institutos, de las asociaciones de gitanos, de los trabajadores sociales y de la CIMADE

13 Esta parte de la investigación es presentada más ampliamente en un artículo publicado en la Revue Ethnologie française escrito en 2008 y titulado « Trajectoires identitaires des Gitans catalans de part et d'autre de la frontière franco-espagnole » en «Sverige-Suède. L'ethnologue dans la cité », Revue Ethnologie Française, pp. 333-343.
} 


\section{Aproximación metodológica: Una antropología de la alteridad}

\section{a) Observación, entrevista, trazados genealógicos...}

Tomando como testigos los hijos originarios de entornos que viven una fuerte alteridad respecto a las poblaciones que les rodean (extranjeros del "exterior" para los magrebíes, en particular los marroquíes de la última ola migratoria, "extranjeros del interior" para los gitanos, sobre todo los catalanes), buscamos comprender qué interacciones de entornos, escolares y familiares, favorecieron el abandono escolar a partir de la afirmación de normas y de valores comunitarios específicos poco favorables a las normas dominantes (por ejemplo las normas escolares o de otras instituciones de la sociedad) que permiten la diversidad social. Además no limitamos la "experiencia de la diversidad social" a las experiencias vividas por los niños únicamente en el contexto escolar, sino también en los distintos entornos a los que pertenecen, en las familias (nucleares, extendidas, clánicas, dispersas o no, ...), y además durante los encuentros de vecindarios nuevos que marcan el despliegue renovado actual de estas poblaciones.

Nuestra aproximación se inscribe, por lo tanto, en una socio-antropología dinámica y compleja de la alteridad, fundada en la renuncia a las prácticas y las posiciones de una etnología endógena a cada población, y en la desnaturalización de los puntos de vista de la institución escolar. Nuestra posición se aleja de los análisis culturalistas o institucionales. Estamos, entonces, interesados en las periferias propicias a las salidas de los territorios "comunitarios", en los lugares y momentos de las interacciones generalizadas que dejan aparecer la alteridad más que la identidad, en los intersticios, en lo que hace que los encuentros sean conflictivos o no, en las situaciones que articulan más fuertemente los territorios de la circulación, es decir alteridades y territorios locales, por lo tanto sedentaridades. Para decirlo de otro modo, a través de este estudio, hemos tratado de volver visibles los factores de "éxito" o de "apañarse" más que el fracaso escolar por si mismo. Hemos tratado de capturar las trayectorias de formación de los jóvenes gitanos y de los migrantes internacionales recién llegados que han resistido a las múltiples dificultades encontradas a lo largo de su escolarización y las respuestas aportadas por las diversas instituciones, particularmente la escuela y la familia. Por lo tanto no se trataba de adoptar un punto de vista normativo considerando por ejemplo que gitanos y marroquíes, recientemente o anteriormente llegados a dichas ciudades, se situarían en alguna etapa de un proceso de integración que les conduciría, después de muchas pruebas, a las conformidades institucionales ciudadanas. 
Nuestros instrumentos, nuestras técnicas de investigación, excluían por lo tanto el cuestionario y las diversas formas objetivadoras en beneficio de entrevistas libres (más de 50 entrevistas semi-dirigidas y 30 entrevistas informales), de la observación directa, el seguimiento y apoyo administrativo, encuentros frecuentes, comentarios de fotografías expuestas o guardadas, de reconstituciones genealógicas, y de sus descripciones en términos de trayectorias socio-espaciales y socio-profesionales. Nuestro trabajo de campo, nuestros interrogantes, nos han mostrado que abordábamos un fenómeno complejo, multiforme $y$, sobretodo, que ha necesitado asociar varios niveles de instrumentos metodológicos: hemos practicado la observación de las disposiciones familiares favorables a la escolarización, la observación de las redes familiares internacionales (marroquíes) o transfronterizas (calificadas de paisanas o de clánicas por los gitanos catalanes), la descripción de los momentos clave de la relación con la escuela así como el análisis longitudinal de 3 o 4 sucesiones generacionales.

Durante la fase de toma de contacto, de impregnación o que podríamos llamar de presentación, el objetivo era entrar en la categorización de los "otros". Esta fase permitió ocupar un lugar y permitir ser identificada por las personas, me convertí en "la que trabaja con los pequeños" y a partir de ahí fue posible introducirse en el grupo, lo que facilitó trabar relaciones de proximidad. A partir de ahí se trató de abordar las poblaciones por los lazos sociales, las redes sociales y no únicamente a partir de marcos estadísticos o categorizaciones exteriores. Dicho de otro modo, no se trataba de abordar las poblaciones estudiadas al azar. Los trabajadores sociales nos permitieron comprender y leer rápidamente las situaciones encontradas. Los trabajadores sociales tienen un importante conocimiento de las vidas de las familias de las personas encontradas. Muy rápido nos informaron sobre el ritmo familiar de las personas, sus redes sociales, sus actividades oficiales o no. En suma son, de algún modo, los informantes.

Hemos usado esta aproximación metodológica a lo largo de la reconstrucción de los trazados genealógicos que proponemos en este artículo. Para recoger información sobre las familias encontradas, fue necesario recurrir a la vez a los archivos del registro civil, pero también y sobre todo llegar a identificar a través de las discusiones con las mujeres, los hombres, y también los vecinos, la historia de los matrimonios tradicionales no declarados, así como la historia de los jóvenes que han abandonado el hogar parental sin estar registrados y los distintos apodos de los apellidos de la familia. Fue necesario cruzar toda la información y transcribirlas lo mejor posible sin desvelar los momentos que querían preservar como pertenecientes únicamente a la memoria del grupo. Era necesario entrar en la 
multitud de sus problemas cotidianos y eso fue lo que nos permitió ir hacia la identificación de los contornos familiares, como si cada dificultad que se presentase hoy hiciese referencia al papel de un pariente, de un antepasado. Esta encuesta genealógica me permitió leer las lógicas de transmisión de competencias prácticas, de competencias socio-económicas más allá de las lógicas de transmisión cultural.

Tal como indicamos anteriormente, otros investigadores en antropología han utilizado este método. Pensamos, ante todo, en los trabajos de Alain Reyniers ${ }^{14}$ [Reyniers, 1992] quien muestra que, en el entorno gitano, las alianzas matrimoniales permiten una fuerte y original diversificación de las profesiones. Explica cómo los gitanos ocupan posiciones sociales diversas ligadas a sus actividades de comercio, de artesanía y también de obreros, funcionarios, dueños de empresa, artistas o intelectuales. Muestra también como la selección de alianzas entre familias o entre clanes son tributarias de la reproducción de dichas posiciones. Pensamos, así mismo, en los trabajos de David Lagunas Arias ${ }^{15}$ [Lagunas Arias, 2005] que analiza la manera en que la comunidad de "los calos de Mataró", gitanos catalanes de origen español, se reproduce como grupo diferenciado a través de las alianzas matrimoniales. Señala el autor la importancia de los proyectos profesionales que prevalecen en buen número de dichas uniones.

\section{b) Análisis interaccionista, análisis por la diversidad}

Autores como Norbert Elias ${ }^{16}$ y Alfred Schultz ${ }^{17}$ hablan de nuestros métodos de trabajo. Nos concentramos en los resultados que nos parecen efectivamente pertinentes. Tratamos de leer el sentido de las situaciones encontradas a través de las interacciones cotidianas y no mediante el análisis cultural de las lógicas de aprendizaje. Adoptamos, por lo tanto, una lógica interaccionista.

También hemos privilegiado el análisis por las situaciones de diversidad, lo que nos ha aparecido igualmente apropiado para explicitar las complejidades de los casos susceptibles de derrumbar nuestros supuestos a priori sobre la naturaleza, la definición misma de las situaciones y de las trayectorias de fracaso. Este concepto

\footnotetext{
14 Alain Reyniers, (1992), « La roue et la pierre. Contribution anthropo-historique à la connaissance de la production sociale et économique des Tsiganes », Tesis, Universidad de Paris V - René Descartes.

15 David Lagunas Arias, (2005), Los tres cromosomas. Odemidad, identidad y parenteso entre los Gitanos catalanes, Editorial Comares, Granada.

16 Elias N, (1993), Engagement et distanciation. Contributions à une sociologie de la connaissance, Paris, Fayard.

17 Schutz A, (1987), Le chercheur et le quotidien. Méridiens Klincksieck.
} 
de diversidad nos permite tener en cuenta diferentes variables, (sexo, edad), pero también movilidades geográficas, movilidades sociales, negociaciones con la institución e iniciativas de unos y otros protagonistas. Situar las trayectorias extranjeras a los modelos normativos, siempre virtuales, como portadores de futuros a veces envidiables, de trayectorias de éxito paradójicas respecto a las ejemplificadas por las instituciones. Es decir que el análisis por la diversidad nos permite identificar trayectorias de éxito diferentes a las habituales y no reconocidas como tales por las instituciones, en particular por las instituciones de socialización como la escuela. Al situarnos en la diversidad como categoría analítica no nos limitamos a las experiencias vividas, sino también a las distintas interacciones entre los grupos, lo que nos permite abordar las poblaciones localizadas por los lazos sociales.

\section{c) Trabajo de campo y poblaciones}

Es necesario precisar la elección de las poblaciones. Varias ubicaciones nos han permitido captar diversas situaciones de movilidad, de sedentariedad y diferentes situaciones de diversidad:

- Perpiñán por un parte, presenta el interés de albergar gitanos catalanes sedentarizados en su centro histórico desde los años 1940, gitanos andaluces, llamados españoles, en tránsito migratorio en las periferias desde hace una decena de años y marroquíes de la migración reciente, domiciliados de forma compacta en viviendas sociales durante los últimos quince años.

- Las poblaciones estudiadas eran particularmente móviles. Hemos extendido nuestras investigaciones, con encuestas extensivas, de Perpiñán a Narbona, Béziers, Montpellier, Nîmes y en Cataluña del sur, sobretodo a Barcelona.

- Por otra parte, Toulouse alberga inmigrantes de diversos orígenes, llegados desde los años 1980, que forman parte de las migraciones clásicas y dominantes de la movilización internacional de la fuerza de trabajo. Hemos observado en los institutos peri-urbanos las dificultades encontradas por jóvenes alumnos recién llegados. 


\section{3) Trayectorias intergeneracionales familiares y transmission transfronteriza de las competencias prácticas y de los aprendizajes escolares}

Para comprender cómo se construyen los tíos (con edades comprendidas de 55 a 70 años), nuestra investigación nos ha llevado a interrogarnos sobre las diferencias de trayectorias escolares entre los hermanos de una misma familia. Cuatro dimensiones principales parecen relevantes: 1) el estatus económico, profesional y cultural de las generaciones anteriores. Entre 1910 y 1950 numerosas familias, incluso extensas, accedieron a las clases medias y medias superiores entonces en constitución en las sociedades francesa y española tanto en Barcelona como en Perpiñán. 2) La selección a menudo transfronterizas de las escuelas y de los institutos, 3) las modalidades de aprendizaje de los oficios, 4) los papeles en las transformaciones de las creencias religiosas en el entorno gitano (particularmente a partir de fin de los años 1970). El estudio de las trayectorias individuales y familiares nos ha permitido entender cómo las interacciones entre los diversos elementos de comprensión pueden llevar a las distinciones de los tíos y sobretodo los dispositivos que los clanes ponen en práctica para perpetuar estas situaciones en las transmisiones intergeneracionales: el aprendizaje en la escuela o en el entorno gitano mismo (autoformación) tiene una gran importancia. Nuestros encuestados nos han informado también sobre las jóvenes personalidades (en la cuarentena) cuyos estatus son cercanos a los de los tíos pero que mantienen una relación muy diferente con el contexto político y religioso local y global.

El estatus transfronterizo de diversas familias ha captado nuestra atención dado que la experiencia de vaivén en el espacio gitano catalán (de Tarragona a Lérida y Perpiñán, por decirlo rápidamente) parece estar reservado a las élites gitanas (profesionales del comercio en particular). Hemos tratado de comprender si existe un lazo entre el mantenimiento de dichas movilidades y una exigencia de aprendizaje de saberes elementales y especializados. Esta hipótesis se ha revelado plausible. Para hacerlo, los trazados genealógicos de clanes transfronterizos fueron determinantes.

Nuestros trazados genealógicos muestran cómo las familias de comerciantes que han accedido a las clases medias y medias superiores en los años 1930, a partir de sólidas inserciones en las actividades comerciales (venta de antigüedades, telas, tapices, cuadros) crean alianzas que les confirman y desarrollan su estatus. Sirva como ejemplo el del antepasado tío (76 años en 2002) quien arregla el matrimonio de su hija mayor (bac +2 en 1967) con un hombre originario de una familia de comerciantes locales sin gran reputación, pero que poseen el mismo apellido. Todos 
los esfuerzos del tío contribuirán desde entonces a su éxito y al de sus hijos, hasta provocar, la unión, en segundas nupcias, de su yerno con una joven (única bachiller en cuatro generaciones) de una familia rural pobre. Esta última familia es absorbida poco a poco por la nueva descendencia del tío. Es notable constatar que esta descendencia, directa, biológica, o por adopción patronímica, provee comerciantes y otros profesionales de alto nivel de escolarización, sin que por ello la familia rural asociada por matrimonio obtenga el beneficio de una revalorización de su estatus. El lazo familiar no es provechoso más que para las personas estrechamente ligadas, al menos en nuestras observaciones.

Se notará también el ejemplo de la "salida por arriba" de una familia nuclear gitana cuyo éxito no tiene que ver con las actividades tradicionales: la pareja profesorenfermera y su hija, estudiante en medicina, han abandonado los territorios y sociabilidades gitanas. Esas "salidas" evidentemente, confirman el temor de las poblaciones gitanas de que sus hijos abandonen la comunidad al ser seducidos por la integración en la sociedad paya.

Es importante evocar la cuestión de la ventaja del contexto social catalano-español respecto al contexto francés. La opinión dominante entre las poblaciones gitanas francesas es, en efecto, que el desarrollo económico y cultural, los éxitos profesionales, son más afirmados en el contexto español que en el francés. Avanzan, como previsto, el hecho que la Federación Catalana de las Asociaciones Gitanas (Barcelona) es mucho más eficaz que la de las Asociaciones Gitanas del Rosellón (Perpiñán). Este razonamiento es en parte erróneo y en parte aceptable: las evoluciones paralelas, en cuatro generaciones, a las que se refieren a los dos trazados genealógicos no permiten identificar diferencias reales en la evolución de los clanes.

Hemos notado también que, en el conjunto de los trazados genealógicos, el nivel de escolarización de las mujeres es mayor, es decir que éstas efectúan generalmente trayectorias de escolarización más largas que las de los hombres. Esto es observable en todas las generaciones y es tanto más cierto en las familias en que los adultos transmiten a sus hijos las competencias prácticas para el éxito comercial. También les afecta más la escolarización de los hijos y en particular de las hijas. Los hombres del linaje se ocupan desde muy temprano de la formación de los hijos para llegar rápidamente a una formación práctica, más profesional. 
Tras haber dado estos elementos de análisis y para leer los trazados genealógicos, los presentamos más abajo de forma sucinta describiendo los enlaces, matrimonios, alianzas escogidas entre las personas implicadas. Hacemos aquí una retranscripción casi narrativa para captar mejor la idea general de las movilidades y de las alianzas.

\section{TRAZADO $\mathrm{N}^{\circ} 1$ o movilidades matrimoniales y territoriales: el apetito transfronterizo}

El trazado genealógico que sigue expone un caso muy clásico de absorción de una familia gitana residente de un lado de la frontera por otra residente al otro lado; en ese caso, se trata de una familia de Perpiñán que ha sido "devorada", en tres generaciones, por un clan barcelonés.

- En 1915, Ingrazia, barcelonesa, es una hija de una familia gitana de comerciantes de telas presente en el gran mercado Sant Antoni del barrio central de Sants. Es viuda de un primer marido fallecido de una enfermedad con quien había tenido $\mathbf{3}$ hijos.

- Hacia los años 1920-1925, dos de los hijos y una nuera fallecen en Valencia durante los combates dado que la familia está comprometida en el bando republicano.

- El tercer hijo es empleado en el comercio de Sant Antoni, desposa una prima y producen un linaje barcelonés con destino incierto: numerosos descendientes son perdidos de vista y hay una retirada generalizada de las actividades comercianles de los abuelos.

- De este matrimonio, podemos decir que Ingrazia no tendrá ninguna posteridad en vistas en el entorno gitano de Barcelona.

- Ingrazia se casa en segundas nupcias con un artesano ebanista de gran reputación de St. Jaques, Manel, considerado desde sus cuarenta años, en Perpiñán, como tío (porque su familia es confirmada por su unión con los barceloneses).

- Con Manel tendrá dos hijos: primero un hijo nacido en 1927 y perdido de vista en 1943: ("Me lo quitaron" dirá designando de modo indistinto a las autoridades franquistas y a las de Vichy). También tienen una hija, Pepita. 
- Pepita se casa con un gitano de Perpiñán aprendiz muy hábil de Manel y además homónimo de éste (también se llama Manel).

- Pepita y Manel tienen dos hijos y una hija.

- La hija se casa, sin descendencia, con un gitano de Perpiñán sin empléo.

- Titet, el mayor, llamado así por su baja estatura, será formado muy joven a la ebanistería de restauración de muebles antiguos y trabajará en el taller de Manel, (que para entonces ha fallecido), sin que por ello aparezca un nuevo tío en la familia.

- El segundo hijo, irá muy pronto a reunirse con la familia de comerciantes de Sant Antoni en Barcelona, pero no trabajará en los mercados.

- Desde entonces, todo sucede como si la familia de Barcelona, que había reafirmado a la de Perpiñán, reclamase a su vez un refuerzo:

- Para ello, Pepe, el tío barcelonés, comerciante de telas asegura la posteridad de su descendencia hasta Andreu, nacido a principios de los años 60, casado con 17 años y excelente comerciante.

- Andreu sólo tendrá un hijo minusválido que no presentará las cualidades necesarias para perpetuar el patrimonio familiar.

- Andreu se divorcia y se vuelve a casar con la hija del Perpiñánés Titet, que se Ilama Andressa.

- Andressa se había casado con el último aprendiz de Manel que había fallecido.

- Andressa y Andreu tienen dos hijos: un hijo, Jaume, quien retoma el comercio de Sant Antoni y extiende sus actividades a los mercados semanales de las grandes ciudades catalanas y se apoya en el vivero familiar para encontrar vendedores, y una hija, Clara, quien con cinco años de estudios superiores en una prestigiosa escuela de comercio de la capital catalana, ha abierto recientemente un centro de gestión fiscal y de negociación comercial con su hermano y otros comerciantes de Sant Antoni. Hoy en día el hermano y la hermana ya han abierto tres tiendas más en Barcelona, una en Gerona, otra en Tarragona y dos en Lérida.

Se ve bien aquí que las fronteras políticas de los payos no tienen gran sentido para el despliegue familiar y clánico de los gitanos catalanes. Es más fácil demostrar que 
las uniones, como recursos internos a los clanes, son de importancia capital para la reproducción de los valores y los saberes.

Esos valores y saberse consisten esencialmente (como lo hemos señalado anteriormente) en el aprendizaje del comercio y el mantenimiento del vínculo familiar. Los niños que, de alguna manera están integrados y confundidos con las poblaciones locales son considerados como demasiado alejados de los valores de la comunidad gitana catalana. En Perpiñán se pueden observar numerosas uniones locales entre familias amenazadas de extinción, de pérdida de saberes, de dispersión de los hijos, etcétera. Parecería que el carácter transfronterizo de los "éxitos" es debido a la visibilización extrema de las alianzas y de sus finalidades.

\section{TRAZADO $\mathrm{N}^{\circ} 2$ o fusiones-consolidaciones: ¿dónde está el buen aliado?}

El segundo trazado genealógico, de alguna manera, invierte la iniciativa familiar desarrollada, en esta ocasión, a partir de un tío de Perpiñán.

- Estebe, nacido en los años 20, huye de Perpiñán durante la guerra y se refugia con una familia gitana mallorquina: en esta isla las poblaciones judías estaban protegidas.

- Estebe se casa con una joven mujer judía ${ }^{18}$ con quien tendrá tres hijas. Trabajará con su familia política en la búsqueda, restauración y comercio de cuadros y de tapices exóticos.

- Recorre España y Portugal y vende sus descubrimientos a redes de aficionados afortunados, o a anticuarios de alto nivel instalados en un barrio céntrico, entre la catedral y la rambla de Colón.

- En 1957 vuelve a Perpiñán con su mujer y tres hijas, compra una casa, continúa sus actividades profesionales extendiéndolas al territorio francés y se distingue rápidamente por la asistencia que aporta a los miembros de la comunidad en apuros.

\footnotetext{
18 Las familias gitanas judías migraron a la isla de Mallorca a finales del siglo XV y lograron salvarse relativamente de las persecuciones hispánicas. No pudimos averiguar si la esposa de Estebe pertenecía a una de esas famillas. Las tres hijas de la pareja fueron bautizadas católicas a su nacimiento.
} 
- Antes de cumplir los cuarenta años se le considera un tío: logra cada vez más notoriedad, en particular cuando, en los años 70 y 80 , rechaza las alianzas ${ }^{19}$ que los representantes locales proponen a los gitanos de Perpiñán.

- Estebe, que ha impuesto su apellido y sus competencias profesionales como inevitables no tiene hijos, por lo tanto no tiene heredero.

- Entonces organiza el encuentro entre su hija Dolcetta y Pere.

- Dolcetta es bachiller en Perpiñán y licenciada en artes plásticas en Barcelona.

- Pere es el segundo hijo de una familia de gitanos de las Baleares, (conocida desde su estancia en Mallorca).

- Estebe dice: «Hacía falta un matrimonio oficial, para que nuestros nombres se confundieran y que mis nietos llevasen plenamente el mío. Pero no fue tan fácil, Dios nos puso a prueba y tuve que esperar una generación más para asegurar mi descendencia »

- En efecto Dolcetta da a sus dos hijas y a su hijo una educación que los distancia de la comunidad gitana. Una, asistente social, se casa con un payo profesor de primaria, la segunda, profesora en un instituto privado de Gerona, se mantiene soltera; el hijo se vuelve profesor de instituto y se casa con una enfermera paya. Su hija hace estudios de medicina. Toda la descendencia de la unión entre Pere y Dolcetta abandona la comunidad gitana y se dispersa entre los barrios payo de diferentes ciudades.

- Estebe (el padre de Dolcetta) dice «He sido castigado por haber querido tenerlo todo: el éxito a la manera de los payos y el reconocimiento de los gitanos. Dios me ha castigado. Dolcetta se ha muerto, tal vez a causa de mis reproches y el alma gitana de los niños ha sido robada por las escuelas de los payos. Yo tenía suficiente para darles, no deberían haber ido a la escuela. (...). Entonces le dije a Pere: 'tu trabajas bien conmigo; lo has aprendido todo y eres mi hijo porque llevas mi nombre. Debemos continuar: te casarás con una barcelonesa de buena familia y formaremos a tus chicos a nuestro oficio. Harán como Dolcetta, sólo irán a la

19 Los votos gitanos, que se concentran en los barrios del centro de la ciudad, son negociados contra una protección, o por explicarlo mejor, una invisibilización de los tráficos de estupefacientes y de sus terribles consecuencias, tal como lo han mostrado A. Tarrius, 1999 y L. Missaoui , 2000. 
escuela para aprender mejor nuestro oficio. Crearemos una familia poderosa en Perpiñán y Barcelona a la vez.

- Después de un año de duelo, Pere se casa con Estrella, viuda sin descendencia de Cargol, que era un trapero apreciado en Barcelona.

- Pere y Estrella tendrán tres hijos : una hija, que se volverá abogada « de la causa gitana » en Barcelona, un hijo, Pablo, que trabajará desde los 12 años con su padre y su abuelo, siguiendo estudios que lo llevarán a una licenciatura de arte medieval en Barcelona, y un segundo hijo que abandonará el entorno familiar de Perpiñán-Barcelona para instalarse como comerciante de postales en Mallorca.

- Pablo y su esposa tendrán una hija y dos hijos: Maria, Jeppe, y Estebe, que también aprendieron el oficio con doce años, comenzaron las grandes giras en España y Francia, y aprendieron la historia del arte en el conservatorio de Barcelona. Residen indiferentemente en Barcelona, Gerona y Perpiñán: la frontera, la causante de la desgracia y el éxito del tío Estebe, nunca ha existido para ellos.

- Estebe transfiere, de hecho, su estatus de tío a Pere y el vasto clan transfronterizo inspira el respeto $y$ el reconocimiento: las visitas a las prisiones, los hospitales, las viudas, la formación de los jóvenes al oficio de trapero, de anticuario, de comerciante ambulante de obras de arte, confieren a ese estatus de tío una dimensión inusual, más allá del clan o de la familia.

El caso de Estebe nos muestra los amplios márgenes de maniobra de que disponen los gitanos de Perpiñán, de Mallorca o de cualquier otro lado para lograr los éxitos familiares, siempre y cuando busquen sus recursos humanos en la multiplicidad de circunstancias que han delimitado par ellos el área cultural histórica catalana. Dicho de otro modo, aquí prima la lengua y los lugares, las familias y los acontecimientos que son identificados por una memoria larga y colectiva: no asistimos a una acumulación de los hechos de memoria, sino a un conocimiento compartido que funciona como un recurso dinámico que autoriza nuevas alianzas, nuevos despliegues económicos, en suma, todo tipo de interacciones productoras de movilizaciones colectivas intergeneracionales ${ }^{20}$. Los éxitos tienen en cuenta la

\footnotetext{
20 La obra de Maurice Halbwachs es evidentemente rica en la aclaración de los hechos de la memoria colectiva. Más que sus primeros trabajos sobre los marcos sociales de la memoria, que sugieren el apilamiento de los recuerdos, el pozo mnésico, preferimos inspirarnos de la Topografía legendaria de los Evangelios en la Tierra Santa. PUF 1941. Se describe la superposición de poblaciones con creencias y normas contrastadas, en la Palestina de los años 30 de forma dinámica: la memoria de los lugares, de
} 
importancia de dotar primero de una auto-formación profesional a los descendientes, y así mantienen la iniciativa del devenir de los suyos. Estamos muy lejos de los esquemas de la intervención institucional. Las instituciones tienen un punto de vista sobre las vivencias de los gitanos ligados a su función de intervención social en situaciones de urgencia. Por ello, a menudo no pueden acceder a una lectura inter-generacional en la que se vuelve evidente lo inteligentes y estratégicas que son las elecciones de que son capaces para asegurar su éxito social, y ello, apoyándose en recursos internos a la comunidad gitana. Por ejemplo, a menudo als instituciones tienen una intención de "inmovilización" de las familias gitanas que es contraria a las fuertes movilidades geográficas y sociales estratégicas que ponen en evidencia las uniones intergeneracionales.

Los gitanos se mueven de un extremo al otro del espacio catalán, de lugar en lugar de sedentariedades seculares. Por lo tanto es útil practicar una lectura de las relaciones comunitarias, urbanas o rurales, siguiendo varios planos, varios niveles de las relaciones entre temporalidades, espacios y familias. También es útil integrar el análisis de los lazos sociales, el análisis longitudinal, en particular de las trayectorias familiares, y sus necesarias monografías.

\section{Para concluir}

Estos dos ejemplos de trazados genealógicos nos llevan a identificar y analizar una cierta autonomización social con la transmisión de las competencias prácticas.

El ejemplo de las familias presentadas con ayuda de los trazados genealógicos indica cómo la familia gitana de Perpiñán y la familia gitana de Barcelona se refuerzan mutuamente aglomerándose en clanes con nuevos contornos, transnacionales, mientras que una lectura limitada por la frontera política entre Francia y España sugiere la desagregación simultánea de una y otra. El lazo entre estas familias es un ejemplo de lo que llamamos el proceso de autonomización, a partir del momento en que el entorno desborda los límites del Estado y sus referencias en términos de pertenencias ciudadanas para fijar su devenir propio. Los territorios de la familia, tal como vemos que se reconstituye, sustituye a aquellos específicos y separados de las solidaridades ciudadanas nacionales. La autonomización del grupo cobra sentido por cómo éste se encarga de la circulación y la autoformación de los jóvenes. ¿Dónde situar las tentativas de la escuela frente diversas. 
a tales recursos tan profundos, singulares y tan extraños a las racionalidades y a las finalidades institucionales? Numerosos tíos se declaran dispuestos, hoy en día, a negociar con las instituciones la formación de sus nietos: es lo que sucede, por ejemplo, y por "lo alto", en el primer trazado genealógico que hemos presentado. El caso de los hijos de Dolcetta, sin embargo, es percibido como un desastre por las familias en el segundo trazado. Las mismas generaciones, los mismos conocidos de escuelas superiores, y resultados muy contrastados del punto de vista del enriquecimiento cultural y económico de la comunidad. Entre ellos prima el mantenimiento de la auto-formación a los saberes de los antepasados. Los gitanos, mediante esta práctica de la auto-formación y mediante la autonomización de los modos de transmisión de los saberes y competencias profesionales en su seno, proponen ellos mismos una solución a su segregación social.

\section{Bibliografía}

Elias, Norbert (1993). Engagement et distanciation. Contributions à une sociologie de la connaissance. Paris, Fayard.

Freeman, Linton C. (2004) The development of social network analysis. Vancouver, Empirical Press.

Grafmeyer, Yves y Danserau, Francine (1998). Trajectoires familiales et espace de vie en milieu urbain. Lyon, Presses Universitaires de Lyon, Coll."Transversales".

Halbwachs, Maurice (1941). Topographie légendaire des Evangiles en Terre Sainte. PUF.

Lagunas Arias, David (2005). Los tres cromosomas. Odemidad, identidad y parenteso entre los Gitanos catalanes. Editorial Comares, Granada.

Missaoui, Lamia (1995). «Généralisation du commerce transfrontalier : petit ici, notable là-bas », REMI, Volume 11, p. 53-75.

Missaoui, Lamia (2000). Gitans et santé de Barcelone à Turin. Ed. Trabucaire.

Missaoui, Lamia y Tarrius, Alain, (2004). « Parole, honneur, mobilité. Les " petits migrants " dans les réseaux mondiaux de l'économie souterraine », en Economies Choisies, Dir. N. Barbe y S. Latouche, Col. Ethnologie de la France, Cahier 20, Ed. de la Maison des Sciences de I'Homme. 
Missaoui, Hasnia-Sonia, con la colaboración de Tarrius, Alain, (2004). «L'autoformation communautaire des jeunes gitans et marocains », en La Déscolarisation sous la direction de D. Glasman y F. Oeuvrard, Édition La Dispute.

Missaoui, Hasnia-Sonia (2005). L'école, le collège : y rester ou en sortir. La construction du potentiel de formation parmi les familles d'enfants gitans et maghrébins de Barcelone à Perpignan, Montpellier et Toulouse. Editorial Trabucaire. Barcelone, Perpignan, 110 p.

Missaoui, Hasnia-Sonia (2005). De l'étranger au citoyen : l'école déstabilisée. Mixités scolaires, mixités familiales et attitudes face à la déscolarisation d'enfants Gitans et Maghrébins. Tesis Université de Toulouse-Le-Mirail, Novembre 2005.

Missaoui, Hasnia-Sonia (2007.2-3), « Usage intermittent de l'école : des temporalités scolaires redéfinies par des enfants gitans et maghrébins nouveauxarrivants », en «les temporalités des populations », Espace, Populations, Sociétés, pp. 385-396.

Reyniers, Alain (1992). La roue et la pierre. Contribution anthropo-historique à la connaissance de la production sociale et économique des Tsiganes. Tesis, Université Paris V René Descartes.

Schutz, Alfred (1987). Le chercheur et le quotidien. Méridiens Klincksieck.

Scott, John (1991). Social Network Analysis: A Handbook. London, Sage.

Simmel, Georg (1998). Les pauvres. Ed. PUF.

Simmel, Georg (1908). «Disgression sur l'étranger». En L'Ecole de Chicago. Naissance de l'écologie urbaine. Sous la direction de Y. Grafmeyer y I. Joseph, Edition Aubier, juin 1998.

Tarrius, Alain (1992), (2ème éd.). Fin de siècle incertain à Perpignan. Drogues, pauvretés, communautés d'étrangers, jeunes sans emploi et renouveau des civilités dans une ville moyenne française. Ed. Trabucaire.

Tarrius, Alain, (1992). Les fourmis d'Europe : migrants riches, migrants pauvres et nouvelles villes internationales. L' Harmattan. 210 p, Paris.

Tarrius, Alain, (2002). La mondialisation par le bas. Les nouveaux nomades des économies souterraines. Balland. 169 p. 2002, Paris. 


\section{Leyenda de los árboles genealógicos}

$\triangle$ : Considerado como Tío

1 : Primeras nupcias

2 : Segundas nupcias

Nivel escolar:

Pr. : Primario

Col. : Secundaria obligatoria

Lyc. : Bachillerato

F.S. : Formación Superior
Estatus profesional:

Sect. ag. : Sector agrícola

$\triangle$ - : Artesano

$\triangle$ : Comerciantes a tiempo completo

$\triangle$ : Comerciantes ocasionales

$\triangle \bigcirc:$ Desempleado 
Árbol genealógico $n^{\circ} 1$

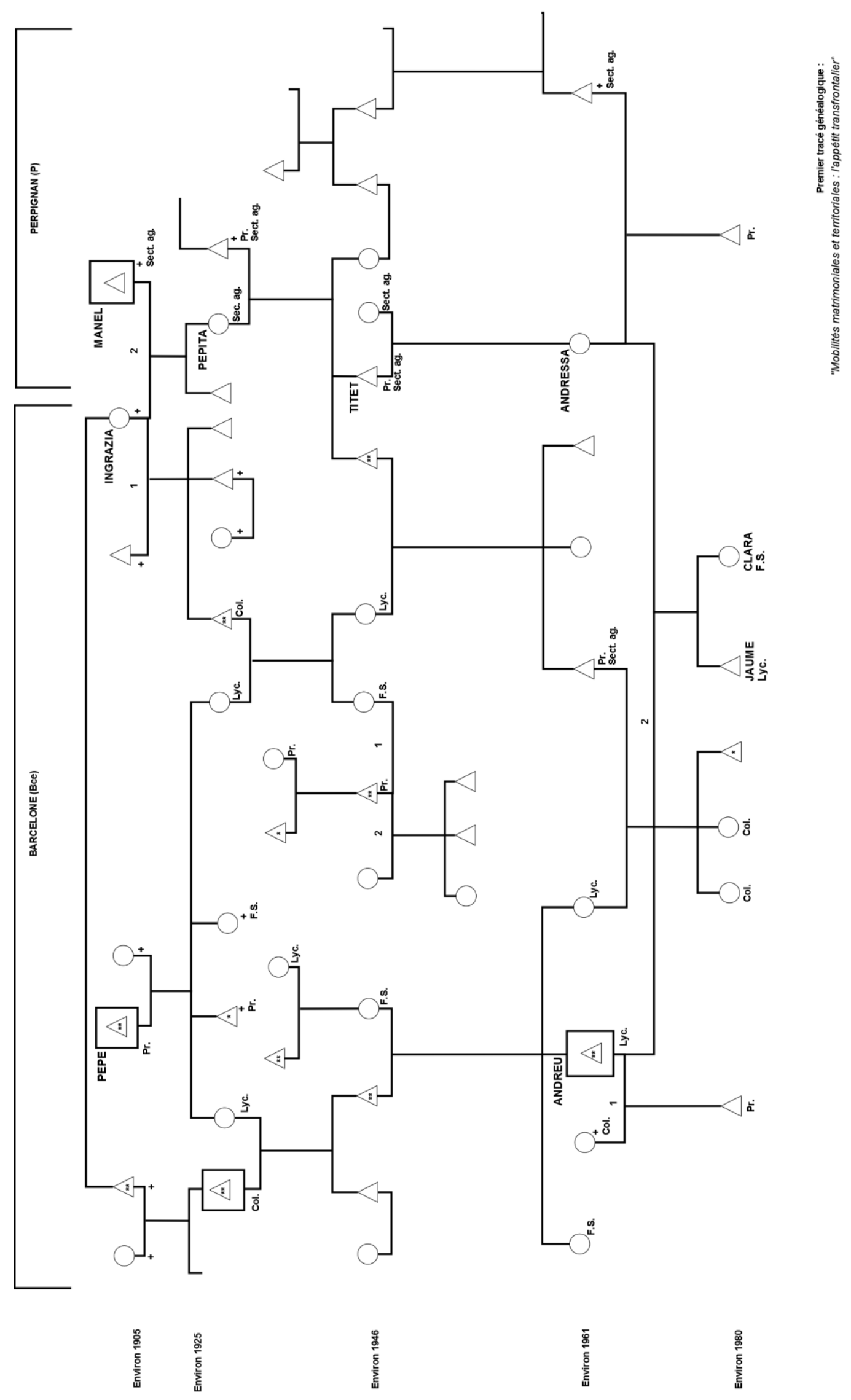




\section{Árbol genealógico $n^{\circ} 2$}

$=$

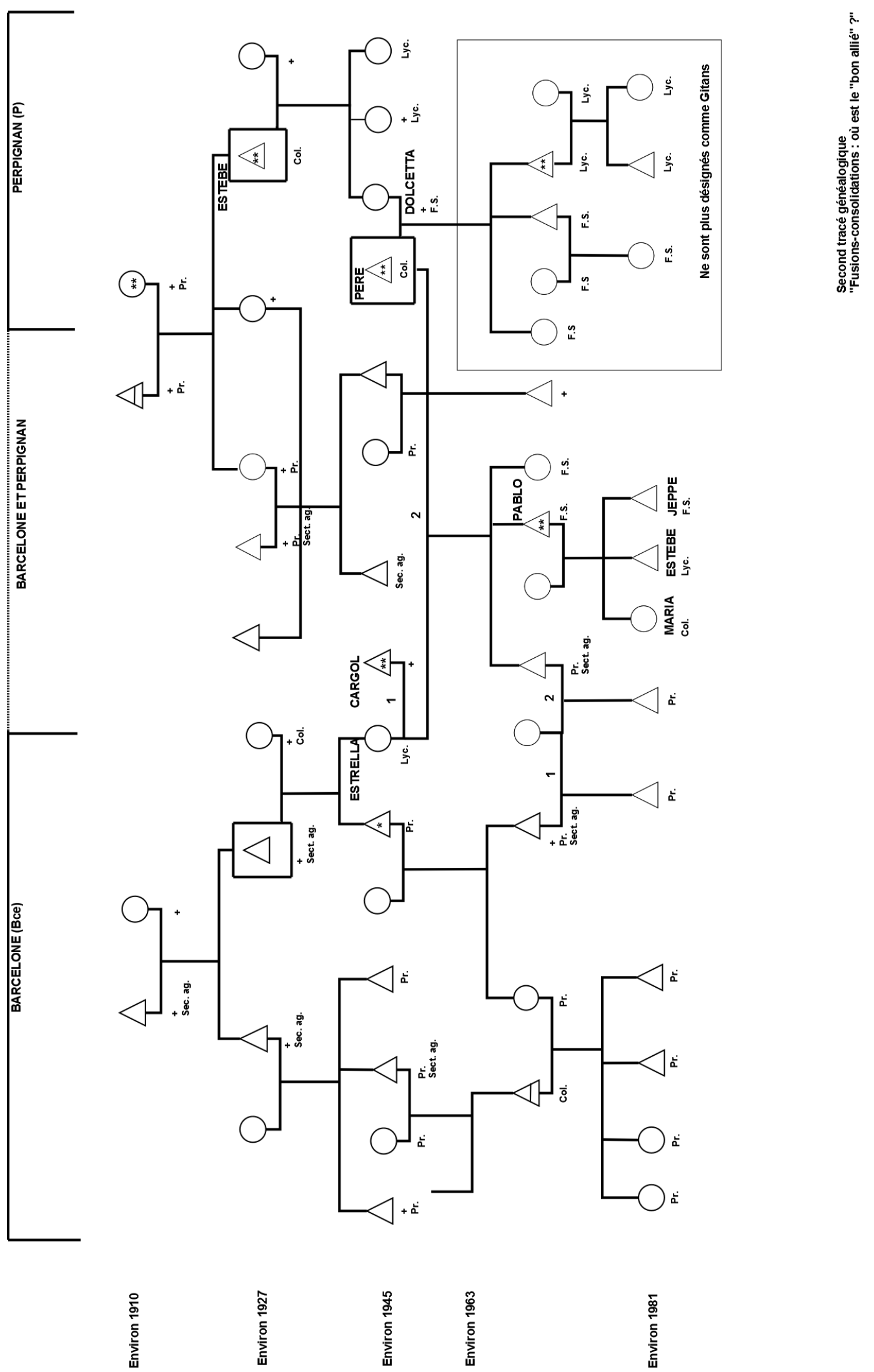

